\title{
Eccentricities of Planets in Binary Systems
}

\author{
Genya Takeda, Frederic A. Rasio \\ Department of Physics and Astronomy, Northwestern University, 2145 Sheridan \\ Road, Evanston, IL 60208, USA
}

September 30, 2005

\begin{abstract}
The most puzzling property of the extrasolar planets discovered by recent radial velocity surveys is their high orbital eccentricities, which are very difficult to explain within our current theoretical paradigm for planet formation. Current data reveal that at least $25 \%$ of these planets, including some with particularly high eccentricities, are orbiting a component of a binary star system. The presence of a distant companion can cause significant secular perturbations in the orbit of a planet. At high relative inclinations, large-amplitude, periodic eccentricity perturbations can occur. These are known as "Kozai cycles" and their amplitude is purely dependent on the relative orbital inclination. Assuming that every planet host star also has a (possibly unseen, e.g., substellar) distant companion, with reasonable distributions of orbital parameters and masses, we determine the resulting eccentricity distribution of planets and compare it to observations? We find that perturbations from a binary companion always appear to produce an excess of planets with both very high $(e \gtrsim 0.6)$ and very low $(e \lesssim 0.1)$ eccentricities. The paucity of near-circular orbits in the observed sample implies that at least one additional mechanism must be increasing eccentricities. On the other hand, the overproduction of very high eccentricities observed in our models could be combined with plausible circularization mechanisms (e.g., friction from residual gas) to create more planets with intermediate eccentricities $(e \simeq 0.1-0.6)$.
\end{abstract}

Keywords: binaries: general — celestial mechanics, stellar dynamics - planetary systems — stars: low-mass, brown dwarfs

\section{Introduction}

As of September 2005, more than 160 extrasolar planets have been discovered by radial-velocity surveys ${ }^{1}$. At least $\sim 10 \%$ are orbiting a component of a wide stellar binary system (Eggenberger et al., 2004). In contrast to the planets in our own solar system, one of the most remarkable properties of these extrasolar planets is their high orbital eccentricities. These high orbital eccentricities are probably not significantly affected by observational selection effects (Fischer and Marcy, 1992). Thus, if we assume that planets initially have circular orbits when they are formed in a disk, there must be mechanisms that later increase the orbital eccentricity. A variety of such mechanisms have been

\footnotetext{
1 For an up-to-date catalog of extrasolar planets, see exoplanets.org or www.obspm.fr/encycl/encycl.html.
}

(c) 2021 Kluwer Academic Publishers. Printed in the Netherlands. 
proposed (Tremaine and Zakamska, 2004). Of particular importance is the Kozai mechanism, a secular interaction between a planet and a wide binary companion in a hierarchical triple system with high relative inclination (Kozai, 1962; Holman et al., 1997; Ford et al., 2000). When the relative inclination angle $i_{0}$ between the orbital planes is greater than the critical angle $i_{\text {crit }}=39.2^{\circ}$ and the semimajor-axes ratio is sufficiently large (to be in a small-perturbation regime), long-term, cyclic angular momentum exchange occurs between the planet and the distant companion, and long-period oscillations of the eccentricity and relative inclination ensue. To lowest order, the maximum of the eccentricity oscillation $\left(e_{1, \max }\right)$ is given by a simple analytic expression:

$$
e_{\max } \simeq \sqrt{1-(5 / 3) \cos ^{2} i_{0}}
$$

(Innanen et al., 1997; Holman et al., 1997). Note that $e_{\max }$ depends just on $i_{0}$. Other orbital parameters, such as masses and semimajor axes of the planet and the companion, affect only the period of the Kozai cycles. Thus, a binary companion as small as a brown dwarf or even another Jupiter-size planet can in principle cause a significant eccentricity oscillation of the inner planet.

Our motivation in this study is to investigate the possible global effects of the Kozai mechanism on extrasolar planets, and its potential to reproduce the unique distribution of observed eccentricities. In practice, we run Monte Carlo simulations of hierarchical triple systems. We have tested many different plausible models and broadly explored the parameter space of such triple systems.

\section{Methods and Assumptions}

The purpose of our study is to simulate the orbits of hierarchical triple systems and calculate the probability distribution of final eccentricities reached by the planet. For each model, 5000 sample hierarchical triple systems are generated, with initial orbital parameters based on various empirically and theoretically motivated distributions, described below. Our sample systems consist of a solar-type host star, a Jupiter-mass planet, and a distant F-, G- or K-type main-sequence dwarf (FGK dwarf) or brown dwarf companion. The possibility of another giant planet being the distant companion is excluded since it would likely be nearly coplanar with the inner planet, leading to very small eccentricity perturbations.

The initial orbital parameters of the triple systems are randomly generated using the model distributions described in Table I. In this 
Table I.

\begin{tabular}{|c|c|c|}
\hline Parameter & Model Distribution Function & Ref. \\
\hline Host-star Mass.... $m_{0}\left(M_{\odot}\right)$ & uniform in $0.9-1.3 M_{\odot}$ & \\
\hline Planet Mass..........m $m_{1}\left(M_{\mathrm{Jup}}\right)$ & uniform in $\log m_{1}, 0.3-10 M_{\mathrm{Jup}}$ & {$[1]$} \\
\hline Secondary Mass... $m_{2}\left(M_{\odot}\right)$ & $\xi\left(q \equiv m_{2} / m_{1}\right) \sim \exp \left\{\frac{-(q-0.23)^{2}}{0.35}\right\}$ & {$[2]$} \\
\hline $\begin{array}{l}\text { Semimajor Axis.... } a_{1}(\mathrm{AU}) \\
\text { of Planet }\end{array}$ & uniform in $\log a_{1}, 0.1-10 \mathrm{AU}$ & {$[1],[3]$} \\
\hline Binary Period........P2 (days) & $f\left(\log P_{2}\right) \sim \exp \left\{\frac{-\left(\log P_{2}-4.8\right)^{2}}{10.6}\right\}$ & {$[2]$} \\
\hline Eccentricity of Planet... $e_{1}$ & $10^{-5}$ & \\
\hline Age of the System... $\tau_{0}$ & uniform in $1-10 \mathrm{Gyr}$ & {$[4]$} \\
\hline
\end{tabular}

[1] Zucker and Mazeh (2002), [2] Duquennoy and Mayor (1991), [3] Ida and Lin (2004), [4] Donahue (1998)

Table II.

\begin{tabular}{lrrrr}
\hline Model & $a_{2, \mathrm{FGK}}(\mathrm{AU})$ & $a_{2, \mathrm{BD}}{ }^{a}(\mathrm{AU})$ & $e_{2}^{b}$ & $\mathrm{BDs}^{c}$ \\
\hline A........ & using $P_{2},<2000$ & $100-2000$ & $10^{-5}-0.99$ & $5 \%$ \\
B........ & using $P_{2},<2000$ & $100-2000$ & $10^{-5}-0.99$ & $10 \%$ \\
C........ & using $P_{2},<2000$ & $100-2000$ & $10^{-5}-0.99$ & $20 \%$ \\
D........ & using $P_{2},<2000$ & $100-2000$ & $10^{-5}-0.99$ & $30 \%$ \\
E........ & & $10-2000$ & $0.75-0.99$ & $100 \%$ \\
F........ & & $10-2000$ & $0.75-0.99$ & $5 \%$ \\
\hline
\end{tabular}

${ }^{a}$ uniform in logarithm

${ }^{b}$ all from thermal distribution, $P\left(e_{2}\right)=2 e_{2}$

${ }^{c}$ the fraction of brown dwarfs in 5000 samples

paper, we present six models, each with different initial conditions that are listed in Table II.

For the calculation of the eccentricity oscillations, we integrated the octupole-order secular perturbation equations (OSPE) derived in Ford et al. (2000). These equations also include GR precession effects, which can suppress Kozai oscillations. As noted by Holman et al. (1997) and Ford et al. (2000), when the ratio of the Kozai period $\left(P_{\mathrm{KOZ}}\right)$, to the GR precession period $\left(P_{\mathrm{GR}}\right)$ exceeds unity, the Newtonian secular perturbations are suppressed, and the inner planet does not experience significant oscillation.

Figure 1 shows typical eccentricity oscillations in two different triple systems. One contains a distant brown dwarf companion and the other a solar-mass stellar companion. The two systems have the same initial 


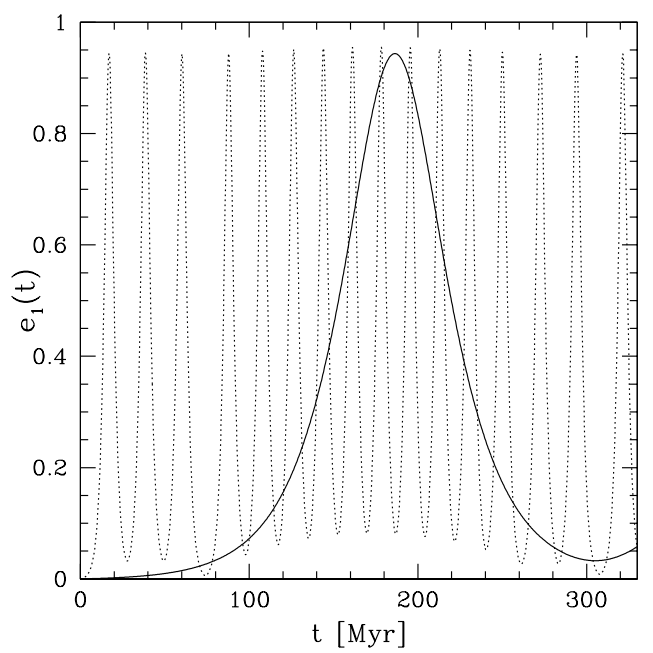

Figure 1. Eccentricity oscillation of a planet caused by a distant brown dwarf companion $\left(M=0.08 M_{\odot}\right.$, solid line) and by a main-sequence dwarf companion ( $M=0.9 M_{\odot}$, dotted line). For both cases, the mass of the planet host star $m_{0}=1 M_{\odot}$, the planet mass $m_{1}=1 M_{\mathrm{J}}$, the planet semimajor axis $a_{1}=2.5 \mathrm{AU}$, the semimajor axis of the companion $a_{2}=750 \mathrm{AU}$, the initial eccentricity of the companion $e_{2}=0.8$, and the initial relative inclination $i_{0}=75^{\circ}$. Note that $e_{1, \max }$ is the same in both cases, as it is dependent only on $i_{0}$, but the smaller mass of a brown dwarf companion results in a much longer oscillation period $P_{\mathrm{KOZ}}$.

orbital inclination $\left(i_{0}=75^{\circ}\right)$, and we see clearly that the amplitude of the eccentricity oscillation is about the same but with a much longer period $P_{\mathrm{KOZ}}$ for the lower mass companion.

To find the final orbital eccentricity distribution, each planetary orbit in our systems is integrated up to the assumed age of the system $\left(\tau_{0}\right)$, and then the final eccentricity $\left(e_{\mathrm{f}}\right)$ is recorded. The results for representative models are compared to the observed eccentricity distribution in $§ 3$. For more details, see Takeda and Rasio (2005).

\section{Results for the Eccentricity Distribution}

Figure 2 shows the final eccentricity in various models. Each model is compared with the distribution derived from all the observed single planets with $a_{1}>0.1$, from the California \& Carnegie Planet Search Catalogue. The statistics of the final eccentricities for our models and for the observed sample are presented in Table 3. Models A-D represent planets in hierarchical triple systems with orbital parameters that are broadly compatible with current observational data and constraints on stellar and substellar binary companions. All the models produce a 


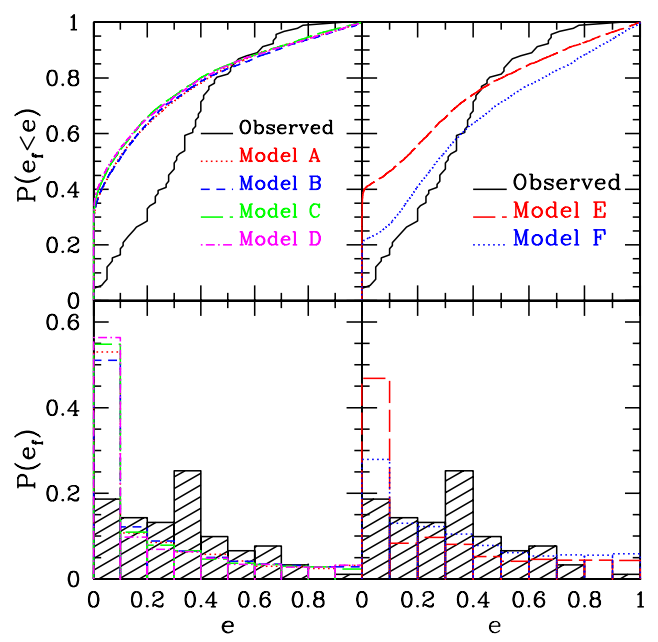

Figure 2. Final cumulative eccentricity distributions (top) and normalized probability distributions in histogram (bottom). Four models with different fractions of brown dwarf and stellar companions. Initial inclinations $\left(i_{0}\right)$ are distributed uniformly in $\cos i_{0}(l e f t)$. Two extreme models where all the binary companions have orbits inclined by more than $40^{\circ}$ (right).

Table III. Statistics of Eccentricity Distributions

\begin{tabular}{|c|c|c|c|c|}
\hline Model & Mean & First Quartile & Median & Third Quartile \\
\hline Observed ......... & 0.319 & 0.150 & 0.310 & 0.430 \\
\hline A.. & 0.213 & 0.000 & 0.087 & 0.348 \\
\hline B... & 0.215 & 0.000 & 0.091 & 0.341 \\
\hline $\mathrm{C} \ldots \ldots \ldots \ldots \ldots \ldots \ldots \ldots \ldots \ldots \ldots$ & 0.201 & 0.000 & 0.070 & 0.322 \\
\hline 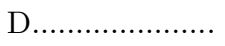 & 0.203 & 0.000 & 0.066 & 0.327 \\
\hline E.... & 0.245 & 0.000 & 0.141 & 0.416 \\
\hline F....................... & 0.341 & 0.071 & 0.270 & 0.559 \\
\hline
\end{tabular}

large excess of planets with $e_{\mathrm{f}}<0.1$ (more than 50\%), compared to only $19 \%$ in the observed sample - excluding multiple-planet systems. An excess of planets which remained in low-eccentricity orbits was evident in most of the models we tested. Changing the binary parameters, such as separations or frequency of brown dwarf companions, did not change this result.

A major difference between most of the simulated and observed eccentricity distributions in the low-eccentricity regime $(e<0.1)$ mainly arises from a large population of binary companions with low orbital 
inclination angle. For an isotropic distribution of $i_{0}$, about $23 \%$ of the systems have $i_{0}<i_{\text {crit }}$, leading to negligible eccentricity evolution. For completeness, biased distributions of $i_{0}$ and $e_{2}$ are tested in model E and $\mathrm{F}$, as an attempt to achieve the best possible agreement with the observations. With all the binary companions having sufficient inclination angles, model $\mathrm{F}$ shows a better agreement with the observed sample in the low-eccentricity regime. However, the number of planets remaining in nearly circular orbit $(e<0.1)$ is still larger than in the observed sample. Moreover, model $\mathrm{F}$ produces the largest excess of planets at very high eccentricities $(e>0.6)$. Note that these extreme models are clearly artificial, and our aim here is merely to quantify how large a bias would be needed to match the observations "at any cost."

\section{Summary and Discussion}

In most of our simulations, too many planets remain at very low orbital eccentricities. The fraction of planets with $e<0.1$ is at least $25 \%$ in our models, but only $\sim 15 \%$ in the observed sample. There are several reasons for this overabundance of low eccentricities in our models. First, the assumption of an isotropic distribution of $i_{0}$ automatically implies that $23 \%$ of the systems have $i_{0}<i_{\text {crit }}$, resulting in no Kozai oscillation. This fraction already exceeds the observed fraction of nearly circular orbits $\left(e_{1}<0.1\right)$ which is $\sim 15 \%$. Systems with sufficient initial relative inclination angles still need to overcome other hurdles to achieve highly eccentric orbits. If many of the binary companions are substellar or in very wide orbits, Kozai periods become so long that the eccentricity oscillations are either suppressed by GR precession, or not completed within the age of the system (or both). This can result in an additional $15 \%-40 \%$ of planets remaining in nearly circular orbits. Even when the orbits of the planets do undergo eccentricity oscillations, there remains still $8-14 \%$ that simply happen to be observed at low eccentricities. Thus, our results suggest that the observed sample has a remarkably small population of planets in nearly circular orbits, and other dynamical processes must clearly be invoked to perturb their orbits. Among the most likely mechanisms is planet-planet scattering in multi-planet systems, which can easily perturb eccentricities to modest values in the intermediate range $\sim 0.2-0.6$ (Rasio and Ford, 1996; Weidenschilling and Marzari, 1996; Marzari and Weidenschilling, 2002). Clear evidence that planet-planet scattering must have occurred in the $v$ Andromedae system has been presented by Ford, Lystad, \& Rasio (2005). Even in most of the systems in which only one giant planet has been detected so far, the second planet could have been ejected as a result of the 
scattering, or it could have been retained in a much wider, eccentric orbit, making it hard to detect by Doppler spectroscopy.

In the high-eccentricity region, where $e_{1} \gtrsim 0.6$, our models show much better agreement with the observed distribution. The Kozai mechanism can even produce a small excess of systems at the highest eccentricities $\left(e_{1}>0.7\right)$, although it should be noted that the observed eccentricity distribution in this range is not yet well constrained. It is evident that the observed planets are rather abundant in intermediate values of eccentricity. The Kozai mechanism tends to populate somewhat higher eccentricities, since during the eccentricity oscillation planets spend more time around $e_{1, \max }$ than at intermediate values. However, this slight excess of highly eccentric orbits could easily be eliminated by invoking various circularization processes. For example, some residual gas may be present in the system, leading to circularization by gas drag (Adams and Laughlin, 2003). In another scenario, decreased periastron distances can consequently remove the orbital energy of the planet by tidal dissipation. This mechanism, referred to as "Kozai migration", was proposed by Wu and Murray (2003) to explain the orbit of HD80606 b. Kozai migration can also circularize the planetary orbit. It is worth to note that the only three massive hot Jupiters in the observed sample, $\tau$ Boo b, GJ86 b and HD195019 b ( $M \sin i>2 M_{\text {Jup }}, P<40$ days) are all in wide binary systems (Zucker and Mazeh, 2002). Their tight orbits with low eccentricity can be a consequence of wider orbits with small periastron distances, initially invoked by the Kozai mechanism.

Clearly, even by stretching our assumptions, it is not possible to explain the observed eccentricity distribution of extrasolar planets solely by invoking the presence of binary companions, even if these companions are largely undetected or unconstrained by observations. However, our models suggest that Kozai-type perturbations could play an important role in shaping the eccentricity distribution of extrasolar planets, especially at the high end. In addition, they predict what the eccentricity distribution for planets observed around stars in wide binary systems should be. The frequency of planets in binary systems is still very uncertain, but the search for new wide binaries among exoplanet host stars has been quite successful in the past few years (e.g., Mugrauer et al, 2005).

\section{Acknowledgements}

We thank Eric B. Ford for many useful discussions. This work was supported by NSF grants AST-0206182 and AST-0507727. 


\section{References}

Adams, F. C. and G. Laughlin: 2003, 'Migration and dynamical relaxation in crowded systems of giant planets'. Icarus 163, 290-306.

Donahue, R. A.: 1998, 'Stellar Ages Using the Chromospheric Activity of Field Binary Stars'. In: ASP Conf. Ser. 154: Cool Stars, Stellar Systems, and the Sun. pp. $1235-+$.

Duquennoy, A. and M. Mayor: 1991, 'Multiplicity among solar-type stars in the solar neighbourhood. II - Distribution of the orbital elements in an unbiased sample'. $A \& A$ 248, 485-524.

Eggenberger, A., S. Udry, and M. Mayor: 2004, 'Statistical properties of exoplanets. III. Planet properties and stellar multiplicity'. A\&A 417, 353-360.

Fischer, D. A. and G. W. Marcy: 1992, 'Multiplicity among M dwarfs'. ApJ 396, 178-194.

Ford, E. B., B. Kozinsky, and F. A. Rasio: 2000, 'Secular Evolution of Hierarchical Triple Star Systems'. ApJ 535, 385-401.

Holman, M., J. Touma, and S. Tremaine: 1997, 'Chaotic variations in the eccentricity of the planet orbiting 16 CYG B.'. Nature 386, 254-256.

Ida, S. and D. N. C. Lin: 2004, 'Toward a Deterministic Model of Planetary Formation. I. A Desert in the Mass and Semimajor Axis Distributions of Extrasolar Planets'. ApJ 604, 388-413.

Innanen, K. A., J. Q. Zheng, S. Mikkola, and M. J. Valtonen: 1997, 'The Kozai Mechanism and the Stability of Planetary Orbits in Binary Star Systems'. AJ 113, 1915-+.

Kozai, Y.: 1962, 'Secular perturbations of asteroids with high inclination and eccentricity'. AJ 67, 591-+.

Marzari, F. and S. J. Weidenschilling: 2002, 'Eccentric Extrasolar Planets: The Jumping Jupiter Model'. Icarus 156, 570-579.

Mugrauer, M., R. Neuhäuser, A. Seifahrt, T. Mazeh, and E. Guenther: 2005, 'Four new wide binaries among exoplanet host stars'. A\& A 440, 1051-1060.

Rasio, F. A. and E. B. Ford: 1996, 'Dynamical instabilities and the formation of extrasolar planetary systems'. Science 274, 954-956.

Takeda, G. and F. A. Rasio: 2005, 'High Orbital Eccentricities of Extrasolar Planets Induced by the Kozai Mechanism'. ApJ 627, 1001-1010.

Tremaine, S. and N. L. Zakamska: 2004, 'Extrasolar Planet Orbits and Eccentricities'. In: AIP Conf. Proc. 713: The Search for Other Worlds. pp. 243-252.

Weidenschilling, S. J. and F. Marzari: 1996, 'Gravitational scattering as a possible origin for giant planets at small stellar distances.'. Nature 384, 619-621.

Wu, Y. and N. Murray: 2003, 'Planet Migration and Binary Companions: The Case of HD 80606b'. ApJ 589, 605-614.

Zucker, S. and T. Mazeh: 2002, 'On the Mass-Period Correlation of the Extrasolar Planets'. ApJl 568, L113-L116. 